\title{
Sistema web personalizable con diseño adaptable para la administración de reservaciones en hoteles
}

\author{
Betzabet García-Mendoza, Carlos R. Jaimez-González \\ Universidad Autónoma Metropolitana, Unidad Cuajimalpa \\ Departamento de Tecnologías de la Información, México \\ 209363465@alumnos. cua.uam.mx, cjaimez@correo.cua.uam.mx
}

\begin{abstract}
Resumen. En este artículo se presenta el diseño e implementación de un sistema web personalizable con diseño adaptable para facilitar a administradores de hoteles pequeños el manejo de reservaciones de sus habitaciones, gestionando las ventas desde su propio sitio web; así como para facilitar y garantizar de inmediato las reservaciones para sus huéspedes potenciales. El sistema web que se presenta utiliza el patrón de diseño web adaptable, por lo que es posible visualizarlo automáticamente en diferentes dispositivos, tales como computadoras de escritorio, laptops, tabletas y teléfonos móviles.
\end{abstract}

Palabras clave: Sistema web personalizable, diseño web adaptable, control de reservaciones, comercio electrónico.

\section{A Customizable Web System with Responsive Design for Administering Hotel Bookings}

\begin{abstract}
This paper presents the design and implementation of a customizable web system with responsive design, for facilitating administrators of small hotels the control of room bookings, managing the sales from their own web site; and facilitates and guarantees the bookings for their potential guests. The web system presented uses the responsive web design pattern in order to be automatically visualized in different devices, such as desktop computers, laptops, tablets and mobile phones.
\end{abstract}

Keywords. Customizable web system, responsive web design, room bookings, e-Commerce.

\section{Introducción}

La planificación de viajes está dominada actualmente por los recursos en línea: agencias de viajes, sitios web de opiniones de viaje, sitios web de opiniones de hote- 
les, redes sociales, sitios web de hoteles, etc. Por ejemplo, el $93 \%$ de los viajeros a nivel mundial dice que sus decisiones de reserva se ven influenciadas por las opiniones en línea [1]. Cuando una persona ha decidido el lugar a visitar, el siguiente paso es buscar dónde hospedarse y dado que el primer medio de búsqueda es el Internet, no hay mejor oportunidad para los hoteles que promocionar sus servicios por Internet. En la actualidad existe una amplia variedad de agencias de viajes, buscadores de hoteles y otras opciones, que permiten localizar hoteles en todo el mundo; sin embargo, la información del hotel que se muestra en estos sitios web es muy escasa y poco útil.

Por otro lado, el $72 \%$ de los viajeros a nivel mundial afirman que la posibilidad de efectuar reservaciones a través de un dispositivo móvil es muy útil, y sólo el 25\% de los alojamientos permite esta funcionalidad [1]. Es por ello que incluir un motor de reservaciones en el sitio web de un hotel es la mejor opción para los hoteles que desean vender de manera fácil y directa, ofreciendo comodidad a sus clientes y abarcando un amplio mercado de ventas: el Internet.

Los sitios web de hoteles tienen como objetivo principal ofrecer el servicio de alojamiento, además de otros servicios generales. Estos sitios se dividen en secciones, donde se describen los diferentes servicios con los que cuenta el hotel, mediante galerías fotográficas, así como secciones de contacto y solicitudes de reservación.

En este artículo se presenta el diseño e implementación de un sistema web personalizable con diseño adaptable para facilitar a administradores de hoteles pequeños el manejo de reservaciones de sus habitaciones, gestionando las ventas desde su propio sitio web; así como para facilitar y garantizar de inmediato las reservaciones para sus huéspedes potenciales. El sistema web que se presenta utiliza el patrón de diseño web adaptable, por lo que es posible visualizarlo automáticamente en diferentes dispositivos, tales como computadoras de escritorio, laptops, tabletas y teléfonos móviles.

El resto del artículo está organizado de la siguiente manera. La sección 2 analiza tres herramientas existentes para personalización de sitios web para hoteles. El análisis y diseño del sistema se presenta en la sección 3, donde se analizan algunos sitios web para hoteles, se realiza un estudio de usuarios, se describe la metodología de desarrollo utilizada para la creación del sistema y se presentan los módulos principales que lo componen. La sección 4 proporciona detalles de la implementación del sistema, con un mapa de navegación, una descripción de las tecnologías y herramientas utilizadas y la implementación del patrón de diseño web adaptable. Las pruebas del sistema y sus resultados se presentan en la sección 5. Finalmente, en la sección 6 se presentan las conclusiones y el trabajo futuro.

\section{Herramientas existentes}

En esta sección se describen tres herramientas que permiten personalizar sitios web para hoteles con motor de reservaciones en línea: PRO Internacional [2], Creowebs [3] y Obehotel [4]. Al final de la sección se presenta una tabla comparativa con características de las herramientas analizadas, la cual se explica detalladamente en [5].

PRO Internacional [2]. De acuerdo a su sitio web, PRO Internacional es una empresa dedicada a brindar servicios web integrales, que van desde la creación de sitios 
web con alto grado de funcionalidad e interactividad, hasta acciones en redes sociales y campañas de posicionamiento en buscadores. Entre los sitios web con alto grado de funcionalidad que crea PRO Internacional, se encuentran los sitios web para hoteles con módulo integrado de reservaciones de habitaciones en línea. Estos sitios web cuentan con las siguientes funcionalidades: reserva de habitaciones en línea, tarifarios por temporadas, galería de fotos, suscripción a boletín electrónico, comentarios, encuestas, buzón de sugerencias. Cabe señalar que PRO Internacional no es una herramienta gratuita, y no cuenta con demostración.

Creowebs [3]. Es una herramienta web que permite crear sitios web a partir de una variedad de plantillas predeterminadas, las cuales están diseñadas para diferentes tipos de sitios web, tales como blogs, sistemas de reservaciones para hoteles o restaurantes, etc. Creowebs proporciona los siguientes módulos para creación de sitios web: plantillas, importación de contenido, editor de imágenes, blog y noticias, redes sociales, videos, música, galerías de fotos, formulario de contacto, compartir archivos, pagos por PayPal, uso de Google AdSense, reservaciones, uso de Google Maps, encuestas, visualización de ruta de navegación y versión para dispositivos móviles.

ObeHotel [4]. De acuerdo a sus creadores, ObeHotel ofrece cuatro diferentes servicios: pasarela de reservaciones; web completa; channel manager; y máxima visibili$d a d$. El servicio de web completa de ObeHotel es el que está relacionado con el sistema web presentado en este artículo, por lo cual fue el que se analizó. Las principales características de web completan son las siguientes: diseño personalizado, creación y configuración de cualquier tipo de habitación, reservación de múltiples habitaciones, gestión de ofertas y paquetes, diferentes tarifas para el sitio web y para los canales de venta, código de rastreo para monitorear visitas, integración con Google Analytics y Google Adwords y módulo web de opiniones. Debido a que el sistema no es gratuito y no cuenta con una demostración para la parte de administración del sistema, no se pudieron probar los módulos correspondientes al administrador.

En la Tabla 1 se muestra una comparación de funcionalidades entre las herramientas analizadas: PRO Internacional, Creowebs y ObeHotel. El símbolo de verificación indica que la herramienta tiene la funcionalidad, mientras que la $x$ que no la tiene. Se proporciona también una descripción de las funcionalidades mostradas en la Tabla 1.

Gestión de habitaciones. Funcionalidad para permitir al administrador la creación, modificación y eliminación de habitaciones.

Reservación múltiple. Funcionalidad para permitir la reservación múltiple en una sola transacción por el mismo huésped potencial.

Tarifas por temporadas. Es la funcionalidad para registrar tarifas por habitación según la temporada del hotel; dichas temporadas son definidas por el administrador con fechas de inicio y fin sin posibilidad de traslapes entre ellas.

Gestión de ofertas y paquetes. Funcionalidad que permite la creación, modificación y eliminación de ofertas y paquetes. Las ofertas son descuentos o promociones en el precio de los servicios; y los paquetes son un grupo de servicios en conjunto.

Mapa de disponibilidad. Esta funcionalidad permite visualizar el mapa de disponibilidad con la relación de habitaciones disponibles y ocupadas. En algunos casos se muestra gráficamente y en otros es una tabla del total de las habitaciones y su estado. 
Comentarios. Funcionalidad para incluir una sección que permita a los huéspedes publicar comentarios sobre su estancia en el hotel.

Encuestas. Funcionalidad que permite al administrador del hotel realizar encuestas de satisfacción de la estancia de los huéspedes.

Buzón de sugerencias. Esta funcionalidad permite incluir un buzón de sugerencias con un formulario, donde el huésped puede emitir una sugerencia al hotel y ésta se envía al correo electrónico del administrador.

Plantillas prediseñadas. Funcionalidad que permite al administrador del hotel elegir una plantilla prediseñada para su sitio web.

Formularios personalizables. Es la funcionalidad que permite al administrador del hotel personalizar formularios; añadiendo y eliminando campos.

Estadísticas. Funcionalidad que permite al administrador conocer información relevante sobre las visitas al sitio web.

Versión móvil. Esta funcionalidad permite visualizar el sitio web desde un dispositivo móvil, ajustando el diseño del sitio web según la resolución del dispositivo.

Tabla 1. Características para evaluar las herramientas analizadas.

\begin{tabular}{lccc}
\hline \multicolumn{1}{c}{ Características } & PRO Internacional & Creowebs & ObeHotel \\
\hline \hline Gestión de habitaciones & $\checkmark$ & $\checkmark$ & $\checkmark$ \\
Reservación múltiple & $\boldsymbol{x}$ & $\checkmark$ & $\checkmark$ \\
Tarifas por temporadas & $\checkmark$ & $\mathbf{x}$ & $\mathbf{x}$ \\
Gestión de ofertas y paquetes & $\boldsymbol{x}$ & $\checkmark$ & $\checkmark$ \\
Mapa de disponibilidad & $\checkmark$ & $\checkmark$ & $\checkmark$ \\
Comentarios & $\checkmark$ & $\checkmark$ & $\checkmark$ \\
Encuestas & $\checkmark$ & $\mathbf{x}$ & $\mathbf{x}$ \\
Buzón de sugerencias & $\checkmark$ & $\checkmark$ & $\mathbf{x}$ \\
Plantillas prediseñadas & $\checkmark$ & $\checkmark$ & $\mathbf{x}$ \\
Formularios personalizables & $\mathbf{x}$ & $\mathbf{x}$ & $\checkmark$ \\
Estadísticas & $\mathbf{x}$ & $\checkmark$ & $\mathbf{x}$ \\
Versión móvil & $\mathbf{x}$ & & $\checkmark$
\end{tabular}

\section{Análisis y diseño}

Esta sección presenta el análisis y diseño llevado a cabo previo a la implementación del sistema web. Se describe la estructura e información proporcionada en sitios web para hoteles; se presenta un estudio de usuarios, donde tres perfiles fueron identificados para el sistema; se muestra la metodología de desarrollo que se utilizó para la creación del sistema; y finalmente, se proporcionan detalles de los módulos principales que componen al sistema web. 


\subsection{Estructura de sitios web de hoteles}

Con el objetivo de conocer la estructura, tipo de información y funcionalidades con las que cuentan los sitios web de hoteles, se visitaron seis sitios web de hoteles ubicados en lugares turísticos de México: 1) Las cúpulas en Malinalco, Estado de México [6]; 2) La casa del laurel en Taxco, Guerrero [7]; 3) El encanto en Bernal, Querétaro [8]; 4) Hotel Malinalco en Malinalco, Estado de México [9]; 5) Casa abierta en Valle de Bravo, Estado de México [10]; y 6) Hotel Cristal en Chignahuapan, Puebla [11]. Un análisis comparativo de la información y características que se ofrecen en cada uno de los sitios web de los hoteles visitados se presenta en [5].

\subsection{Estudio de usuarios}

Los perfiles de usuario describen las características de los usuarios del sistema y permiten diseñar un sistema centrado en las necesidades de los usuarios. El estudio de usuarios que se llevó a cabo consistió en identificar a los usuarios del sistema web, mediante el diseño de una encuesta para cada uno, con preguntas que permitieron obtener información sobre sus intereses, necesidades, y características, las cuales se tomaron también en cuenta para el diseño del sistema web resultante.

Se identificaron dos tipos de usuarios: administrador y huésped potencial. Además, se definió un perfil de hotel, debido a que el sistema web se planteó para hoteles con características específicas. Se realizaron un total de 16 encuestas a administradores de hoteles ubicados en los pueblos mágicos de Huasca de Ocampo y Real del Monte, ambos pertenecientes al estado de Hidalgo.

También se realizaron un total de 84 encuestas a turistas que se encontraron en estos pueblos mágicos. Una vez realizadas las encuestas, se analizaron los resultados obtenidos y se procedió a definir los perfiles de usuario, los cuales se utilizaron para el diseño del sistema.

\subsection{Metodología de desarrollo}

Para la realización del sistema web se utilizó una metodología iterativa e incremental, la cual consiste en realizar varias iteraciones, con las siguientes etapas en cada una de ellas: análisis, diseño, implementación y pruebas. Esta metodología es incremental ya que en cada iteración se agregan nuevas funcionalidades al sistema. Con esta metodología fue posible obtener de manera inicial un prototipo funcional desde la primera iteración, y posteriormente fue posible evaluar con el cliente dicho prototipo para obtener retroalimentación y resolver problemas de manera temprana.

A través del desarrollo iterativo e incremental se logra una versión más estable del sistema al final de cada iteración, la cual tiene mayor calidad y posee nuevas funcionalidades con respecto a versiones anteriores. Para este proyecto se realizaron cuatro iteraciones, agregando nuevos módulos al sistema hasta completar la funcionalidad total del mismo; se hicieron adecuaciones de acuerdo a la retroalimentación recibida del cliente, y se corrigieron los errores detectados en las pruebas de las iteraciones anteriores. 


\subsection{Módulos del sistema}

Los requerimientos generales para el sistema web fueron obtenidos del hotel Bosque Mágico, el cual fue uno de los hoteles con los que se tuvo contacto en la etapa de estudio de usuarios. Se eligió este hotel debido a la variedad de opciones y servicios que ofrece, lo cual permitió tener un sistema más general y completo. A partir de los requerimientos y del análisis previo realizado, se determinó que el sistema web estaría compuesto de cuatro módulos principalmente.

Módulo de Edición. Permite al administrador personalizar el portal web con las características de su hotel. El administrador proporciona información al sistema acerca de habitaciones, paquetes, servicios, reservaciones, entre otros.

Módulo de Administración. Permite al administrador manejar las reservaciones del hotel, ya que se tiene acceso a la información de cada una. El administrador es capaz de visualizar a través de un calendario, la disponibilidad del hotel.

Motor de Reservaciones. Este módulo permite al huésped potencial y al administrador verificar la disponibilidad del hotel, proporcionando fecha de ingreso, fecha de salida y número de personas. También es posible reservar una o más de las habitaciones mostradas en la lista de disponibilidad.

Portal Web. Permite al huésped potencial navegar a través del portal que fue personalizado por el administrador a través del módulo de edición. En este portal web el huésped potencial puede realizar reservaciones y encontrar información acerca de paquetes, habitaciones, servicios, atracciones, ubicación del hotel, entre otros.

Para cada uno de estos módulos se llevaron a cabo los correspondientes casos de uso, modelo de interfaz, diagramas de clases y diagramas de secuencia, los cuales fueron utilizados en cada iteración con el administrador del hotel, para obtener retroalimentación y llegar a acuerdos en el desarrollo del sistema.

\section{Implementación}

La implementación del sistema web se presenta en esta sección. Se muestra el mapa de navegación del sistema con los dos usuarios del sistema: administrador y huésped potencial; se proporcionan las tecnologías y herramientas utilizadas para el desarrollo del sistema; y finalmente, se describe la implementación del patrón de diseño web adaptable, el cual permitió adaptar la interfaz del sistema web a diferentes dispositivos para mejorar su visualización y navegación.

\subsection{Mapa de navegación}

La Figura 1 muestra el mapa de navegación del sistema, con los dos usuarios del sistema: el administrador del hotel y el huésped potencial. El mapa de navegación muestra el conjunto de páginas web a las cuales tiene acceso cada usuario.

El administrador puede acceder dos secciones principalmente: la edición del portal y la administración. En la edición del portal el administrador puede personalizar el portal web mediante la creación, modificación y eliminación de habitaciones, temporadas, servicios, paquetes, promociones, información de contacto, galerías fotográfi- 
cas y atracciones. En la sección de administración, el administrador puede manejar todas las reservaciones, realizar reservaciones manualmente en el sistema, modificar reservaciones existentes, visualizar reservaciones en el mapa de disponibilidad de habitaciones y desplegar estadísticas del sistema.

El huésped potencial puede acceder a todas las páginas web personalizadas por el administrador: servicios, atracciones, galerías fotográficas, información de contacto, promociones, habitaciones, paquetes y reservaciones de habitaciones y paquetes. Para realizar una reservación de habitación o paquete, el huésped potencial puede verificar la disponibilidad de habitaciones en la página web de reservaciones.

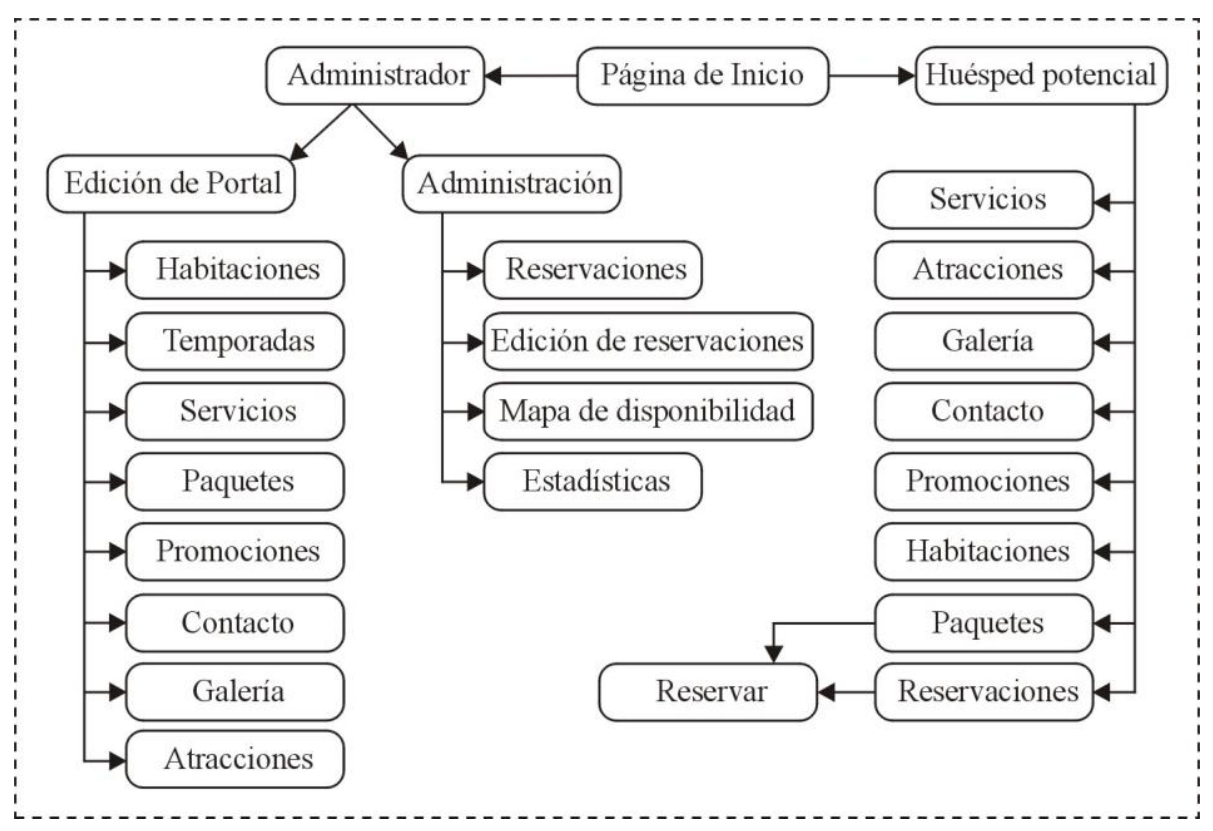

Fig. 1. Mapa de navegación del sistema.

\subsection{Tecnologías y herramientas utilizadas}

Para el desarrollo del sistema web se utilizaron una serie de tecnologías y herramientas, algunas de las cuales se mencionan a continuación.

Java Server Pages (JSP). Es una tecnología Java que permite el desarrollo de páginas web dinámicas, combinando HTML estático con HTML generado dinámicamente. Los archivos JSP fueron utilizados para la creación de páginas web dinámicas, donde la información se extrae de una base de datos y se procesa.

JavaScript. Es un lenguaje de programación interpretado, diseñado para hacer páginas web interactivas y dinámicas. JavaScript fue principalmente utilizado para la creación dinámica de páginas web, especialmente para la estructura dinámica de la información que se despliega en los diferentes dispositivos. 
Cascading Style Sheets (CSS). Es un lenguaje de estilos utilizado para presentar información en una página web. El mecanismo para cambiar la posición de elementos HTML en una página web es ampliamente utilizado para organizar la información cuando se accede al sistema web desde diferentes dispositivos, debido a que el espacio de visualización disponible en cada dispositivo puede ser diferente.

NetBeans. Es un ambiente de desarrollo integrado, el cual permite escribir, compilar, depurar y ejecutar programas. NetBeans permitió concentrar todos los archivos del proyecto, tales como clases Java, archivos JSP y JavaScript, entre otros.

Apache Tomcat. Es un servidor web y contenedor de servlets, el cual permite compilar archivos JSP que son convertidos en servlets. Apache Tomcat fue utilizado para el procesamiento de los archivos JSP del sistema web.

MySQL. Es un sistema manejador de bases de datos relacionales, basado en el lenguaje de consulta estructurado (Structured Query Langauge, SQL por sus siglas en inglés). MySQL fue utilizado para la creación de la base de datos del sistema web, así como para ejecutar sentencias tales como SELECT, INSERT, UPDATE, entre otras.

\subsection{Diseño web adaptable}

El diseño web adaptable (Responsive Web Design, RWD por sus siglas en inglés) $[12,13]$ es un patrón de diseño que permite adaptar la interfaz de un portal web a diferentes dispositivos con el objetivo de mejorar la visualización y la experiencia de navegación de los usuarios. Actualmente, hay una gran variedad de dispositivos móviles con diferentes resoluciones y tamaños de pantalla, lo cual es la principal razón por la que el diseño de interfaces web requiere adaptarse a esos dispositivos. RWD está basado en Media Queries, los cuales son una serie de instrucciones en CSS que definen ciertas características de anchura, altura, orientación, color y otros parámetros del dispositivo desde el cual el portal web es accedido. Esto permite aplicar diferentes estilos para cada dispositivo mediante la determinación de su tamaño de pantalla o resolución, de tal manera que sea posible tener diferentes vistas del mismo portal web.

Debido a que actualmente hay diferentes dispositivos desde los que un sitio web puede ser accedido, el sistema web presentado en este artículo define tres vistas para ser visualizado: una vista para teléfonos móviles, una vista para tabletas y una vista para computadoras de escritorio y laptops. La forma más frecuente de distribuir la información es a través de un número específico de columnas de acuerdo a la vista; por ejemplo, una columna para la vista de teléfonos móviles, dos columnas para la vista de tabletas y tres columnas para la vista de computadoras de escritorio y laptops. La Figura 2 muestra un ejemplo de cómo puede ser arreglada la distribución de información en columnas de acuerdo al dispositivo.

Cabe señalar que en algunos casos los Media Queries no son lo suficientemente flexibles para organizar información. Este es el caso del sistema web presentado en este artículo, en el cual cierta información es creada dinámicamente y la cantidad de elementos a ser visualizados en el portal web es variable. Por esta razón, en este sistema fue necesario adicionalmente la implementación de varias funciones JavaScript, las cuales permitieron la organización de la información de acuerdo al dispositivo desde el cual es portal web es accedido. Las funciones JavaScript creadas fueron con- 
troladas utilizando los atributos window.innerWidth y window.innerHeight, los cuales permiten conocer el tamaño de la ventana del navegador web, de tal forma que se puede determinar el número de columnas que será utilizado para dividir la información y ser visualizada. Las funciones JavaScript creadas son ejecutadas cuando se disparan los eventos onresize y onload del navegador web.

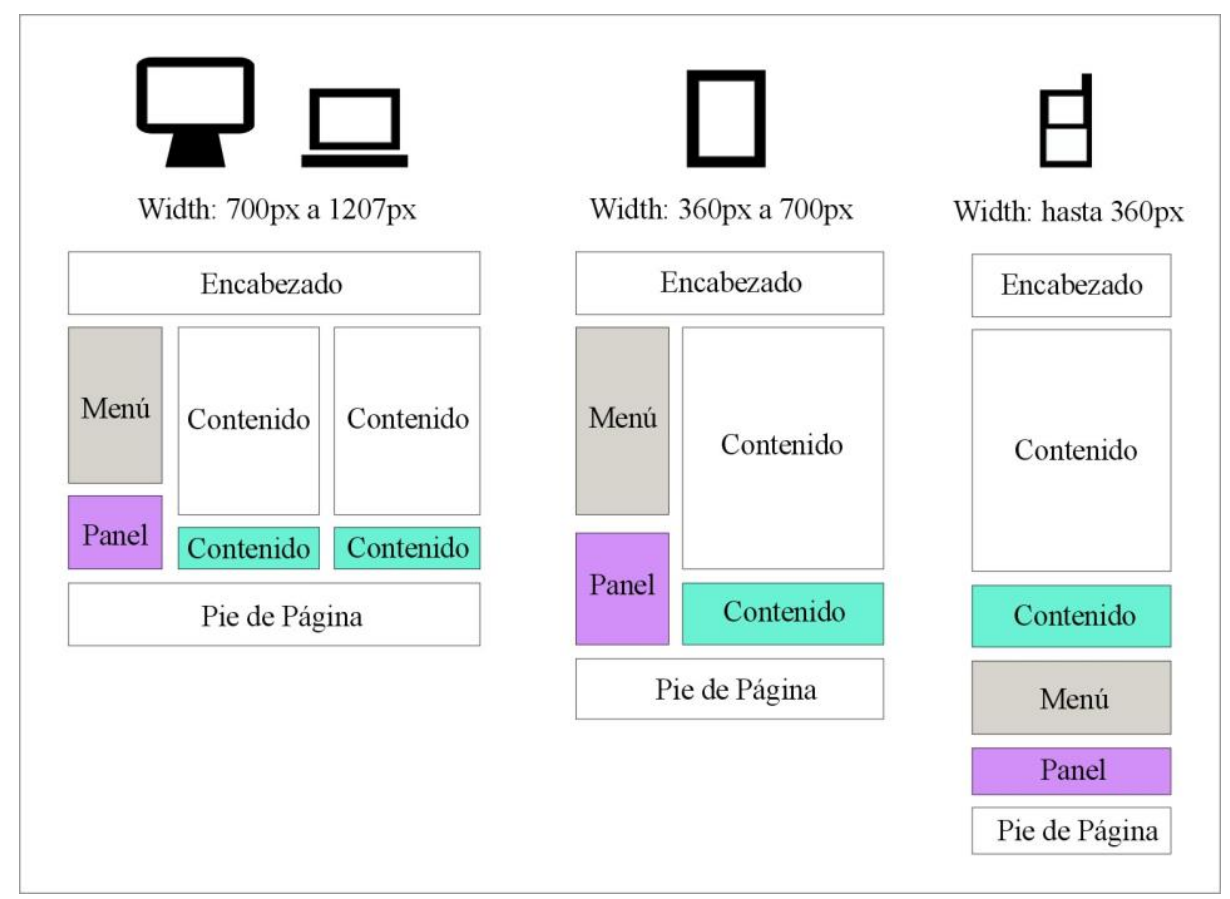

Fig. 2. Distribución de información en columnas de acuerdo al dispositivo.

\section{Pruebas y resultados}

Esta sección presenta las pruebas llevadas a cabo en el sistema y sus resultados. Se realizaron dos tipos de pruebas: de funcionalidad y de interfaz.

Las pruebas de funcionalidad fueron realizadas a través de controles de prueba para determinar el éxito o fracaso del sistema en tareas específicas, las cuales fueron parte del levantamiento de requerimientos con el usuario y plasmadas como casos de uso que se realizaron en la etapa de análisis y diseño de la metodología de desarrollo. Los resultados de estas pruebas fueron los siguientes: $92 \%$ de los controles de prueba fueron exitosos, mientas que el $8 \%$ de ellos falló. Cabe señalar que las pruebas fallidas estuvieron relacionadas con tareas donde las fechas de reservaciones se traslapaban y con precios que estaban equivocados de acuerdo a las temporadas.

Las pruebas de interfaz se llevaron a cabo para verificar la correcta visualización del portal web en los diferentes dispositivos para los cuales fue diseñado: teléfono móvil, tableta, laptop y computadora de escritorio. La Tabla 2 muestra los dispositi- 
vos en los que el portal web fue probado. Las columnas de la tabla indican la siguiente información acerca del dispositivo: tipo, marca, modelo, sistema operativo, navegador web, resolución de la pantalla en pixeles. También se muestran las capturas de pantalla de la visualización del sistema web con las habitaciones disponibles en un hotel en tres diferentes dispositivos de los que se utilizaron en las pruebas (1, 3 y 5).

Tabla 2. Dispositivos utilizados en las pruebas de interfaz.

\begin{tabular}{ccccccc}
\hline Tipo & $\#$ & Marca & Modelo & S.O. & Navegador & Resolución \\
\hline \hline Laptop & 1 & Dell & XPS - M1330 & Windows & Chrome & $1280 \times 800$ \\
Laptop & 2 & HP & Pavilion 15p213ns & Windows & Chrome & $1366 \times 768$ \\
Tableta & 3 & Apple & iPad mini 2 & iOS 8 & Safari & $768 \times 1024$ \\
Tableta & 4 & Samsung & Galaxy Tab 4 & Android & Chrome & $800 \times 1280$ \\
Teléfono & 5 & Alcatel & One touch & Android & Android & $320 \times 568$ \\
Teléfono & 6 & Samsung & Galaxy S4 & Android & Android & $360 \times 640$ \\
\hline
\end{tabular}

La Figura 3 muestra la interfaz web desplegada en una laptop con una resolución de 1280 por 800 pixeles. La Figura 4 muestra del lado izquierdo la interfaz web desplegada en una tableta con una resolución de 768 por 1024 pixeles, y en el lado derecho muestra la misma interfaz web desplegada en un teléfono móvil con una resolución de 320 por 568 pixeles.

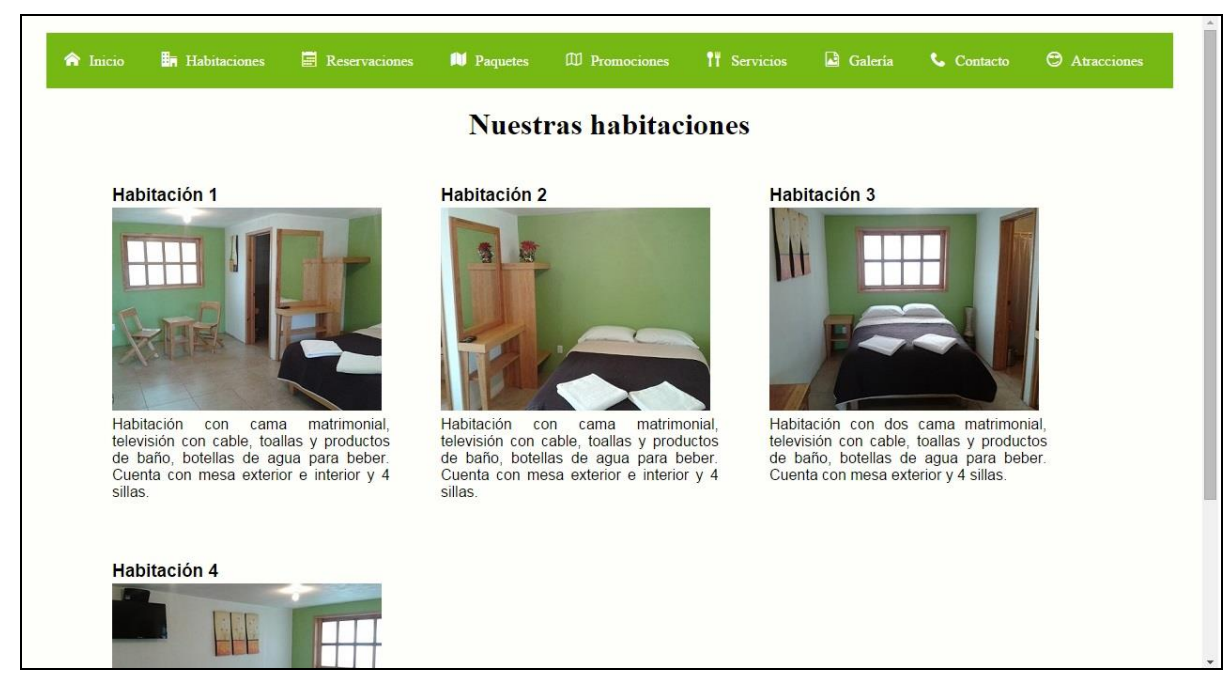

Fig. 3. Sistema web desplegado en una laptop (1280x800).

La página web de la Figura 3 está dividida en dos paneles: el panel superior, el cual tiene el menú con diferentes opciones para el huésped potencial; y el panel inferior, el cual es utilizado para desplegar el contenido de la página y contiene las habitaciones disponibles organizadas en tres columnas. 
La captura de pantalla de la Figura 4 (izquierda) muestra la misma página web desplegada en una tableta, con información reducida y menú simplificado. La información de las habitaciones disponibles es organizada en dos columnas, el menú está oculto en la parte superior y se visualiza del lado izquierdo cuando el usuario da click sobre el icono ubicado en la esquina superior derecha. La Figura 4 (derecha) es la misma página web, pero desplegada en un teléfono móvil, en la que las habitaciones están organizadas en una sola columna y el menú está también oculto.
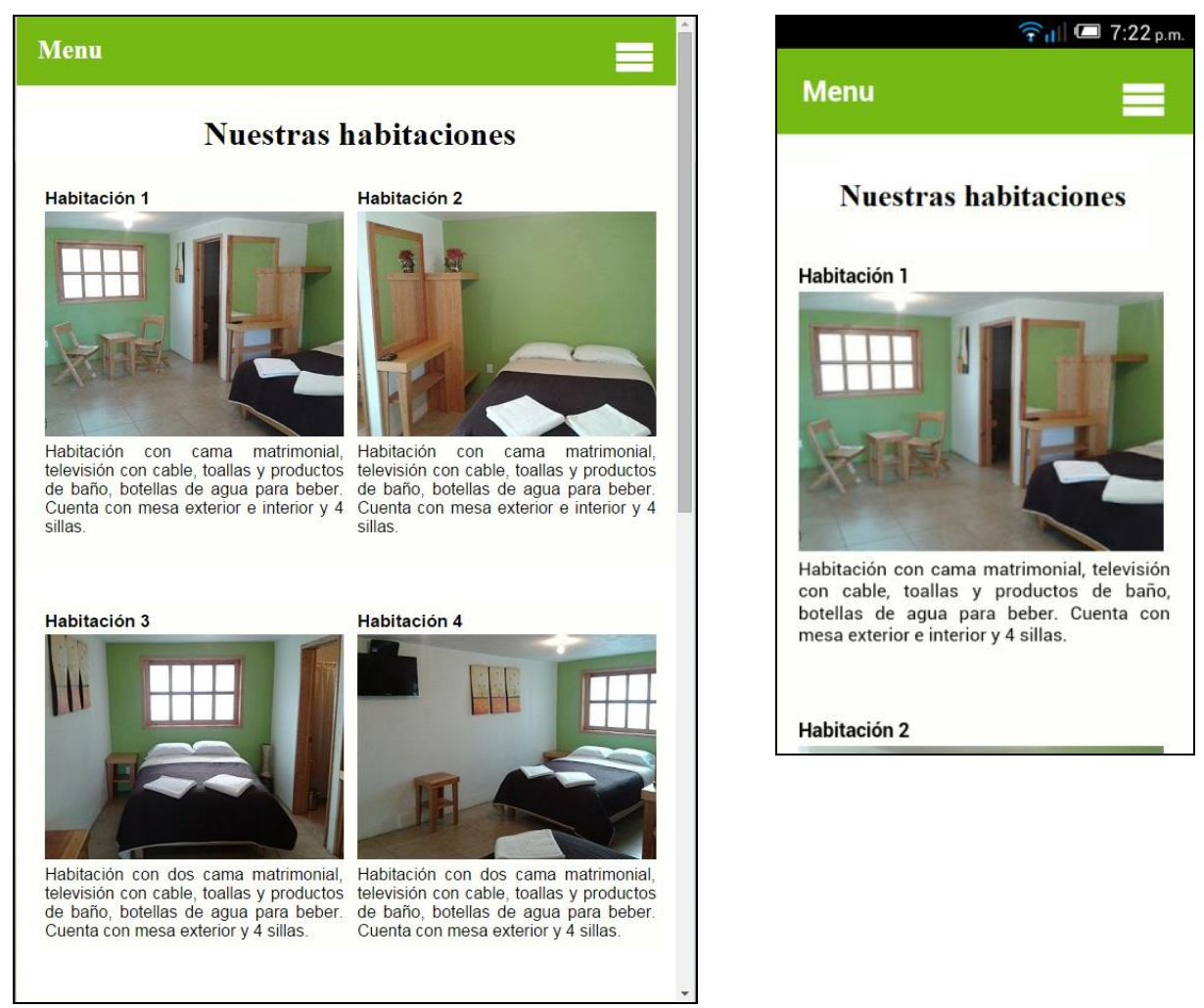

Fig. 4. Sistema web desplegado en una tableta (768x1024) y en un teléfono móvil (320x568).

\section{Conclusiones y trabajo futuro}

En este artículo se presentó el diseño e implementación de un sistema web personalizable con diseño adaptable, el cual cumplió con los requisitos planteados al inicio de su desarrollo, así como con las necesidades de los clientes, las cuales se tomaron a partir del levantamiento de requerimientos y de las encuestas realizadas. El sistema web facilita a administradores de hoteles pequeños el manejo de reservaciones de sus habitaciones, gestionando las ventas desde su propio sitio web; así como facilita y garantiza de inmediato las reservaciones para sus huéspedes potenciales. 
Se logró exitosamente que el sistema web se adaptara automáticamente a diferentes dispositivos, tales como computadoras de escritorio, laptops, tabletas y teléfonos móviles, utilizando el patrón de diseño web adaptable, mediante el uso de CSS, Media Queries y JavaScript. Esta característica de adaptación se verificó a través de las pruebas de interfaz que se realizaron sobre diferentes dispositivos. En particular, los resultados se mostraron mediante capturas de pantalla de las interfaces desplegadas en tres dispositivos: en una laptop con una resolución de 1280 por 800 pixeles, en una tableta con una resolución de 768 por 1024 pixeles, y en un teléfono móvil con una resolución de 320 por 568 pixeles.

Es necesario llevar a cabo pruebas de usabilidad con administradores de hoteles, así como con huéspedes potenciales. El sistema será ajustado después de las pruebas realizadas y se pondrá en funcionamiento en los hoteles que cumplan con el perfil de hotel definido en el estudio de usuarios.

\section{Referencias}

1. Trip Barometer: Encuesta realizada a hoteleros y viajeros. Disponible en: http://www.tecnohotelnews.com/wp-content/uploads/2013/03/In fografia TripBarometer.jpg

2. Sitio web de PRO Internacional. Disponible en: http://www.prointernacional. $\mathrm{com} /$

3. Sitio web Creowebs. Disponible en: https://creowebs.com/

4. Sitio web ObeHotel. Disponible en: http://www . obehotel.com/

5. García-Mendoza, B., Jaimez-González, C. R.: Propuesta de Sistema Web Personalizable para el Control de Reservaciones en Hoteles. Research in Computing Science: Advances in Intelligent Information Technologies, 79, 135-145 (2014)

6. Sitio web del hotel Las cúpulas. Disponible en: http://lascupulas.com.mx/

7. Sitio web del hotel La casa del laurel. Disponible en: http://www. hotelentax co.com/es/index.html

8. Sitio web del hotel El encanto. Disponible en: http://elencantobernal. $\mathrm{com} / \mathrm{acerca}$

9. Sitio web del hotel Hotel Malinalco. Disponible en: http://www. hotelmalinal $\mathrm{co} . \mathrm{com} \cdot \mathrm{mx} /$

10. Sitio web del hotel Casa abierta. Disponible en: http://www. casabierta.com. $\mathrm{mx} /$ index.html

11. Sitio web del hotel Hotel Cristal. Disponible en: http://www.hotelcristal chignahuapan.com.mx/

12. Tendencias en diseño web. Disponible en: http://www.tecnohotel news.com/2013/07/las-tendencias-de-futuro-en-diseno-webpara-hoteles/

13. Responsive Web Design. Disponible en: http://www.designtribe. ie/news/24/61/New-Responsive-B-B-Hotel-website 